\title{
Systematic review of research design and reporting of imaging studies applying convolutional neural networks for radiological cancer diagnosis
}

\author{
Robert J. O'Shea ${ }^{1}$ (i) - Amy Rose Sharkey ${ }^{1,2} \cdot$ Gary J. R. Cook ${ }^{1,3} \cdot$ Vicky Goh $^{1,2}$ \\ Received: 10 October 2020 / Revised: 24 February 2021 / Accepted: 12 March 2021 / Published online: 16 April 2021 \\ (C) The Author(s) 2021
}

\begin{abstract}
Objectives To perform a systematic review of design and reporting of imaging studies applying convolutional neural network models for radiological cancer diagnosis.

Methods A comprehensive search of PUBMED, EMBASE, MEDLINE and SCOPUS was performed for published studies applying convolutional neural network models to radiological cancer diagnosis from January 1, 2016, to August 1, 2020. Two independent reviewers measured compliance with the Checklist for Artificial Intelligence in Medical Imaging (CLAIM). Compliance was defined as the proportion of applicable CLAIM items satisfied.

Results One hundred eighty-six of 655 screened studies were included. Many studies did not meet the criteria for current design and reporting guidelines. Twenty-seven percent of studies documented eligibility criteria for their data (50/186, 95\% CI 21$34 \%$ ), 31\% reported demographics for their study population (58/186, 95\% CI 25-39\%) and 49\% of studies assessed model performance on test data partitions (91/186, 95\% CI 42-57\%). Median CLAIM compliance was 0.40 (IQR 0.33-0.49). Compliance correlated positively with publication year $(\rho=0.15, p=.04)$ and journal H-index $(\rho=0.27, p<.001)$. Clinical journals demonstrated higher mean compliance than technical journals $(0.44$ vs. $0.37, p<.001)$.

Conclusions Our findings highlight opportunities for improved design and reporting of convolutional neural network research for radiological cancer diagnosis.

\section{Key Points}

- Imaging studies applying convolutional neural networks (CNNs) for cancer diagnosis frequently omit key clinical information including eligibility criteria and population demographics.

- Fewer than half of imaging studies assessed model performance on explicitly unobserved test data partitions.

- Design and reporting standards have improved in CNN research for radiological cancer diagnosis, though many opportunities remain for further progress.
\end{abstract}

Keywords Artificial intelligence $\cdot$ Deep learning $\cdot$ Diagnosis, computer-assisted $\cdot$ Neoplasms $\cdot$ Research design

Robert J. O'Shea

robert.1.oshea@kcl.ac.uk

1 Cancer Imaging, School of Biomedical Engineering and Imaging Sciences, King's College London, 5th floor, Becket House, 1 Lambeth Palace Road, London SE1 7EU, UK

2 Department of Radiology, Guy's \& St Thomas' NHS Foundation Trust, London, UK

3 King's College London \& Guy's and St. Thomas' PET Centre, London, UK

\section{Abbreviations}

AI

CLAIM

$\mathrm{CNN}$

CONSORT

EQUATOR

IQR

MI-CLAIM

PRISMA
Artificial intelligence

Checklist for AI in Medical Imaging Convolutional neural network Consolidated Standards of Reporting Trials Enhancing the Quality and Transparency Of health Research Interquartile range Minimum Information about Clinical Artificial Intelligence Modelling Preferred Reporting Items for Systematic reviews and Meta-Analyses 
QUADAS Quality Assessment Tool for Diagnostic Accuracy Studies

SPIRIT-AI Standard Protocol Items: Recommendations for Interventional Trials-Artificial Intelligence extension

STARD Standards for Reporting of Diagnostic Accuracy Studies

STARD-AI Standards for Reporting of Diagnostic Accuracy Studies - Artificial Intelligence extension

STROBE Reporting of Observational studies in Epidemiology
Following ImageNet 2012 [22], convolutional neural network (CNN) models have been adapted to various biomedical tasks. The approach is now the industry standard in AI applications for diagnostic radiology [23, 24]. In this study, we aim to quantify explicit satisfaction of the CLAIM criteria in recent studies applying CNNs to cancer imaging. We examine the adequacy of data and ground truth collection, model evaluation, result reporting, model interpretation, benchmarking and transparency in the field. We identify key areas for improvement in the design and reporting of $\mathrm{CNN}$ research in the field of diagnostic cancer imaging.

\section{Materials and methods}

\section{Inclusion criteria}

1. The article evaluates a $\mathrm{CNN}$ model for radiological cancer diagnosis in humans.

\section{Introduction}

Recent years have seen an increase in the volume of artificial intelligence (AI) research in the field of cancer imaging, prompting calls for appropriately rigorous design and appraisal standards [1-6]. Evaluation of AI research requires a skillset which is distinct from those of classical medical statistics and epidemiology. The problems of high dimensionality, overfitting and model generalisation are central challenges in AI modelling [7-10]. These phenomena potentially compromise the generalisation of AI models to the reality of clinical practice [11]. However, the reliability of these models may be estimated and maximised through rigorous experimental design and reporting $[1,12]$.

EQUATOR was founded to improve the quality of scientific research through standardisation of reporting guidelines $[13,14]$. Established EQUATOR guidelines such as STARD [15], STROBE [16] and CONSORT [17] were not designed specifically to address the challenges of AI research. AIfocused guidelines have recently been developed including CLAIM [18], SPIRIT-AI [19], MI-CLAIM [20] and, prospectively, STARD-AI [21]. These are welcome measures as AI remains at an early phase of clinical implementation for diagnostic tasks. Although each set of reporting standards addresses a specific task, a high degree of overlap exists between these guidelines, reflecting the fundamental importance of many of the criteria.

CLAIM aims to promote clear, transparent and reproducible scientific communication about the application of AI to medical imaging and provides a framework to assure highquality scientific reporting. Current conformity to these standards has not been formally quantified to date. Consequently, a need exists for a contemporary evaluation of design and reporting standards in the domain of cancer imaging AI research.
2. The model receives a radiological image as its sole input.

3. The article was published in a peer-reviewed journal between January 1, 2016, and August 1, 2020.

4. The article is published in the English language.

\section{Exclusion criteria}

1. The model addresses a non-diagnostic task such as preprocessing, segmentation or genotyping.

2. The model receives non-radiological images such as histopathology, dermoscopy, endoscopy or retinoscopy.

3. The article presents experiments on animal or synthetic data.

4. The article primarily addresses economic aspects of model implementation.

5. The article is published in a low-impact journal.

6. The article is unavailable in full-text format.

\section{Search}

PubMed, EMBASE, MEDLINE and SCOPUS databases were searched systematically for original articles from January 1, 2016, to August 14, 2020, for articles meeting our inclusion and exclusion criteria. Search queries for each database are included in the supplementary material. The search was performed on August 14, 2020. No other sources were used to identify articles. Screening and decisions regarding inclusion based on the full text were performed independently by 2 reviewers (R.O.S., A.S., clinical fellows with 3 years and 1 year of experience of AI research, respectively) and disagreements resolved by consensus. A senior reviewer (V.G.) was available to provide a final decision on unresolved 
Table 1 List of the data items evaluated. Items are derived from the CLAIM guidance. CLAIM items with multiple conditions are divided into sub-items, denoted as alphabetical suffixes. Compliant values are all values considered satisfactory for that item. Exemptions indicate types of study which are not required to satisfy an item

\begin{tabular}{|c|c|c|c|c|}
\hline Item & Criterion & Values & $\begin{array}{l}\text { Compliant } \\
\text { values }\end{array}$ & Exemptions \\
\hline 1 & $\begin{array}{l}\text { Title or abstract specified application of } \\
\text { convolutional neural network model }\end{array}$ & $\begin{array}{l}0 . \text { Not specified } \\
\text { 1. Specified }\end{array}$ & 1 & None \\
\hline 2 & $\begin{array}{l}\text { Abstract included summary of study design, } \\
\text { methods, results and conclusions }\end{array}$ & $\begin{array}{l}0 . \text { Not included } \\
\text { 1. Included }\end{array}$ & 1 & None \\
\hline 3 & $\begin{array}{l}\text { Introduction provided scientific and clinical } \\
\text { background with role for model }\end{array}$ & $\begin{array}{l}\text { 0. Not provided } \\
\text { 1. Provided }\end{array}$ & 1 & None \\
\hline $4 \mathrm{a}$ & Study objectives & $\begin{array}{l}0 . \text { Not provided } \\
\text { 1. Provided }\end{array}$ & 1 & None \\
\hline $4 b$ & Study hypotheses & $\begin{array}{l}0 . \text { Not documented } \\
\text { 1. Documented }\end{array}$ & 1 & None \\
\hline 5 & $\begin{array}{l}\text { Indicated prospective or retrospective study } \\
\text { timeframe }\end{array}$ & $\begin{array}{l}0 . \text { Not documented } \\
\text { R. Retrospective } \\
\text { P. Prospective } \\
\text { RP. Both retrospective and prospective }\end{array}$ & $\mathrm{R}, \mathrm{P}, \mathrm{RP}$ & None \\
\hline 6 & Study goal & $\begin{array}{l}0 . \text { Not documented } \\
\text { 1. Documented }\end{array}$ & 1 & None \\
\hline $7 \mathrm{a}$ & Data source & $\begin{array}{l}0 . \text { Not documented } \\
\text { L. Local data collection } \\
\text { P. Public data } \\
\text { LP. Both local and public data }\end{array}$ & $\mathrm{L}, \mathrm{P}, \mathrm{LP}$ & None \\
\hline $7 b$ & Data collection institutions & $\begin{array}{l}0 . \text { Not documented } \\
\text { SC. Single-centre data } \\
\text { MC. Multi-centre data }\end{array}$ & $\mathrm{SC}, \mathrm{MC}$ & None \\
\hline $7 \mathrm{c}$ & Imaging equipment vendors & $\begin{array}{l}0 . \text { Not documented } \\
\text { SV. Single vendor } \\
\text { MV. Multiple vendors }\end{array}$ & SV, MV & None \\
\hline $7 \mathrm{~d}$ & Image acquisition parameters & $\begin{array}{l}\text { 0. Not documented } \\
\text { 1. Documented }\end{array}$ & 1 & None \\
\hline $7 \mathrm{e}$ & Institutional review board approval & $\begin{array}{l}0 . \text { Not documented } \\
\text { 1. Documented }\end{array}$ & 1 & None \\
\hline $7 f$ & Participant consent & $\begin{array}{l}0 . \text { Not documented } \\
\text { 1. Documented }\end{array}$ & 1 & None \\
\hline 8 & Eligibility criteria & $\begin{array}{l}\text { 0. Not documented } \\
\text { 1. Documented }\end{array}$ & 1 & None \\
\hline 9 & Image pre-processing & $\begin{array}{l}\text { 0. Not documented } \\
\text { P. Pre-processing documented } \\
\text { PM. Reproducible pre-processing method doc- } \\
\text { umented } \\
\text { NP. Documented that pre-processing not } \\
\text { employed }\end{array}$ & PM, NP & None \\
\hline 10 & Data subsetting & $\begin{array}{l}\text { 0. Not documented } \\
\text { C. Image cropping documented } \\
\text { CM. Reproducible image cropping method } \\
\text { documented } \\
\text { NC. Documented that cropping not employed }\end{array}$ & $\mathrm{CM}, \mathrm{NC}$ & None \\
\hline 11 & Model predictors and outcomes & $\begin{array}{l}0 . \text { Not defined } \\
1 . \text { Not defined }\end{array}$ & 1 & None \\
\hline 12 & Data de-identification & $\begin{array}{l}\text { 0. Not documented } \\
\text { A. Anonymisation documented } \\
\text { AM. Reproducible anonymisation method } \\
\quad \text { documented }\end{array}$ & $\mathrm{AM}$ & None \\
\hline 13 & Missing data handling strategy & $\begin{array}{l}0 . \text { Not documented } \\
\text { E. Missing data excluded from analysis } \\
\text { I. Missing data included in analysis }\end{array}$ & E, I & None \\
\hline 14 & Reference standard definition & $\begin{array}{l}\text { 0. Not defined } \\
\text { 1. Defined either explicitly or by reference to a } \\
\text { Common Data Element such as the } \\
\text { American College of Radiology Image } \\
\text { Reporting and Data Systems. }\end{array}$ & 1 & None \\
\hline
\end{tabular}


Table 1 (continued)

Item Criterion

15a Reference standard rationale

$15 b$

Definitive ground truth

Manual image annotation

16b Histopathology annotation

17 Image annotation tools and software

18 Annotator variability

19a Sample size

19b Provided power calculation

19c Distinct study participants

20 Data partitions and their proportions

21 Partition disjunction

22a Provided reproducible model description

22b Provided source code

23 Modelling software

24 Parameter initialisation method

Provided reproducible data augmentation strategy or specified used of unaugmented data

$25 \mathrm{~b}$ Loss function

25c Optimisation method

25d Learning rate settings

25e Stopping protocol for model training

$25 \mathrm{f}$ Batch size
Values

0 . Not documented

1. Documented

0 . No definitive ground truth

P. Histopathology

DI. Definitive imaging modality

FU. Case follow-up

PFU. Histopathology and case follow-up

PDI. Histopathology and definitive imaging modality

0 . Not documented

UR. Radiologist with unspecified expertise

SR. Radiologist with relevant subspecialist expertise

OC. Other clinician

0 . Not documented

SP. Pathologist with relevant subspecialist expertise

0 . Not documented

1. Documented

0 . Not documented

V. Variability statistics documented

M. Aggregation method documented

VM. Variability statistics and aggregation method documented

0 . Not documented

1. Documented number of images in dataset

0 . Not documented

1. Documented

0 . Not documented

$\{\mathrm{N}\} . \mathrm{N}=$ number of study participants

0 . Not documented

1. Documented

0 . Not documented

1. Documented partition disjunction at patient level

0 . Not documented

1. Documented

0 . Not documented

1. Documented

0 . Not documented

S. Documented software

$\mathrm{SV}$. Documented software and version

0 . Not documented

R. Random initialisation

T. Transfer learning

RT. Both random initialisation and transfer learning employed

0 . Not documented

Compliant

Exemptions

values

$\begin{array}{ll}1 & \text { None } \\ \text { P, DI, FU, PFU, None } \\ \text { PDI }\end{array}$

SR

None

SP

Histopathology not employed

1

None

VM

None

1

None

1

None

$\{\mathrm{N}\}$

None

1

None

1

Validation studies

1

Validation studies

1

SV

Validation studies

A. Documented data augmentation

AM. Reproducible data augmentation method NA. No data augmentation

0 . Not documented

1. Documented

0 . Not documented

1. Documented

0 . Not documented

1. Documented

0 . Not documented

1. Documented

0 . Not documented

1. Documented
AM, NA

Validation studies

Validation studies

Validation studies

Validation studies

Validation studies

Validation studies 
Table 1 (continued)

Item Criterion

26 Model selection

27 If model ensembling applied, provided ensembling method

$28 \quad$ Metrics

29 Significance

30 Robustness

31 Model interpretation

32 Test data description

33 Case-flow diagram

34 Demographics and clinical characteristics

35a Test performance

35b Human diagnostic performance benchmarking

35c Computational diagnostic performance benchmarking

36 Diagnostic performance with measure of precision

$37 \quad$ Failure analysis

38 Study limitations

39 Clinical implications of study findings

40 Study registration number
Values

Compliant

Exemptions

values

0 . Not documented

1. Documented model selection criterion, specifying $k$ if $k$-fold cross validation employed

0 . Not documented

E. Ensembling documented

EM. Documented reproducible ensembling method

0 . Not documented

1

Validation studies

M. Defined performance metrics

MR. Defined performance metrics and provided rationale

0 . Not documented

None

S. Model significance documented

SM. Model significance documented with reproducible methodology

0 . Not documented

1

None

1. Documented model robustness to variation in experimental conditions such as sample size, noise and imaging equipment

0 . Not documented

IM

Ensembling not employed

I. Interpreted model

IM. Interpreted model with validated methodology

0 . Not described

I. Employed internal test data

E. Described test data from different institution

0 . Not documented

1. Documented

0 . Documented

D. Documented aggregate statistics

DP. Documented statistics for each data partition

0 . Model performance assessed on data observed during training

V. Model performance assessed on data observed during model selection

T. Model performance assessed on data which was unobserved during training and model selection

0 . No human performance benchmark

None

UR. Benchmarked against radiologist with unspecified expertise

SR. Benchmarked against radiologist with relevant subspecialist expertise

OC. Benchmarked against other clinicians

0 . No computational benchmark

I, E

None

None

None

DP

$\mathrm{T}$

None

1. Benchmarked against other computational methods

0 . Diagnostic performance reported without measure of precision

1. Diagnostic performance reported with confidence interval or standard error

0 . Not discussed 1

1. Discussed misclassified cases or model errors

0 . Not discussed

1. Discussed

0 . Not discussed

1. Discussed

0 . Not documented

1. Documented 
Table 1 (continued)

\begin{tabular}{lllll}
\hline Item & Criterion & Values & $\begin{array}{l}\text { Compliant } \\
\text { values }\end{array}$ & Exemptions \\
\hline 41 & Study protocol & & 1 & None \\
42 & \multirow{2}{*}{ Funding } & 0. Not documented & 1. Provided access to the full study protocol & \\
& & 0. Not documented & FR, NF & None \\
& F. Funding source documented & & \\
& FR. Funding source and role documented & & \\
& NF. Stated no funding received & \\
\hline
\end{tabular}

disagreements. Duplicated articles were removed. Articles were not screened with the QUADAS tool [25], as it shares several items with the CLAIM guideline. Exclusion of QUADAS-incompliant articles would have biased subsequent estimations of CLAIM compliance.

\section{Data extraction}

Data items were defined to measure compliance with CLAIM proposal and previously published proposals $[1,18]$. Complex items with multiple conditions were subdivided as appropriate. Data items are listed in Table 1. First author, journal, publication year, modality and body system were also extracted. Studies which served to validate existing models were exempt from all items pertaining to model development. Studies not employing model ensembling were exempt from item 27.

Articles were read and annotated by R.O.S. and A.S., and disagreements were resolved by consensus. Articles were read in random order, using a fixed sequence generated in R [26]. Journal H-index was extracted from the Scimago journal rankings database [27]. Journals were categorised as either "clinical" or "technical" according to the journal name-names containing any term related to computer science, artificial intelligence or machine learning were assigned the "technical" category. The remaining journals were assigned the "clinical" category.

\section{Data analysis}

Statistical analysis was conducted using R version 3.5.3 [26] and RStudio version 1.1.463 [28]. For each item, the proportion of compliant studies was measured, excluding those with applicable exemptions. For items with $\geq 3$ response categories, proportions were also measured for each category. Ninety-five percent confidence intervals $(95 \% \mathrm{CI})$ were estimated around binary proportions using the method of Clopper and Pearson [29] and around multinomial proportions using the method of Sison and Glaz [30, 31]. Following adherence assessment recommendations [32], an overall CLAIM compliance score was defined per article by the proportion of applicable items satisfied. Items and subitems were weighted equally.

$$
\text { CLAIM compliance }=\frac{\text { number of items satisfied }}{\text { number of items applicable }}
$$

Temporal change in CLAIM compliance was evaluated by two-sided test of Spearman rank correlation between CLAIM score and year of publication. Association between journal impact factor and compliance was evaluated with a twosided test of Spearman rank correlation between journal Hindex and CLAIM score. The difference in mean CLAIM compliance between clinical and technical journals was evaluated with a two-sided $t$ test. All code and data required to support the findings of this research are available from the corresponding author upon request. As a methodological review assessing study reporting, this study was not eligible for registration with the PROSPERO database.

\section{Results}

\section{Search}

Six hundred fifty-five articles were identified in the primary database search, of which 267 were duplicates. One hundred twenty articles were excluded during title screen, and 82 articles were excluded during abstract screening. One hundred eighty-six articles were included in the final analysis. A flow diagram for the literature search process is provided in Fig. 1. The dataset included articles from 106 journals. Fifty-four clinical journals and 44 technical journals were included. Assigned journal categories are provided in Supplementary Table 1. The distributions of article publication year, body system and modality for are provided in Fig. 2.

\section{Title, abstract and introduction}

Compliance for items $1-13$ is shown in Fig. 3. Ninety-one percent of studies identified their model as a convolutional neural network $(170 / 186,95 \%$ CI $86-95 \%)$ and $70 \%$ 

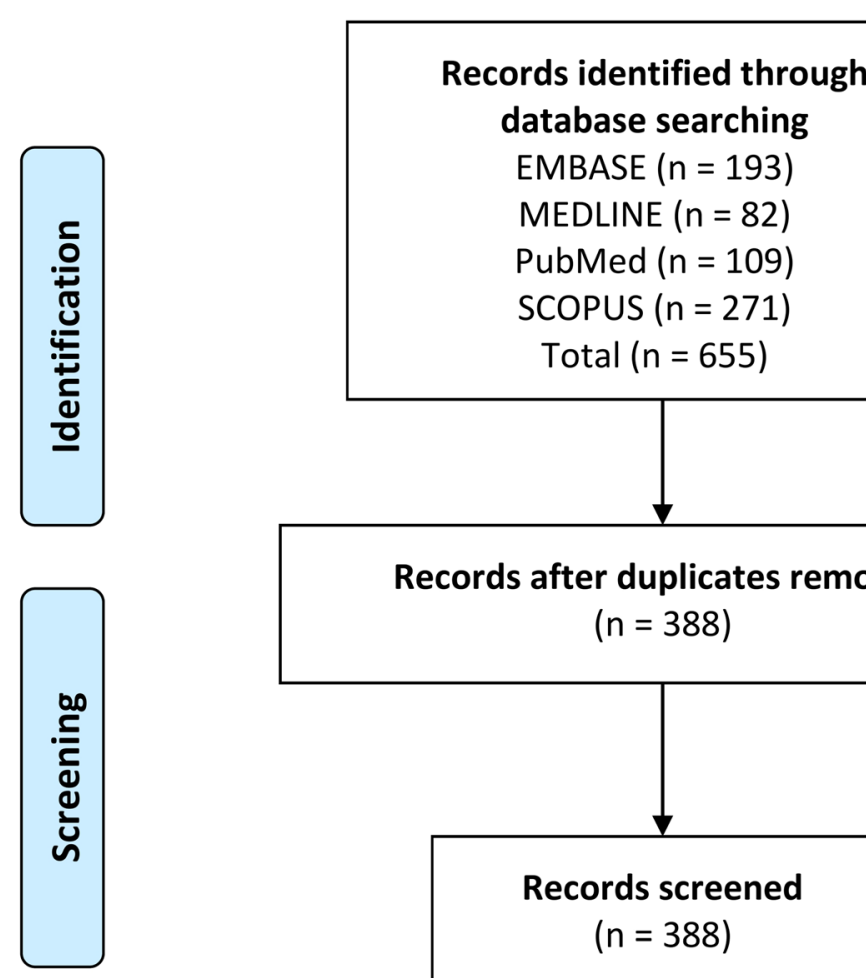

\section{Records after duplicates removed} $(n=388)$
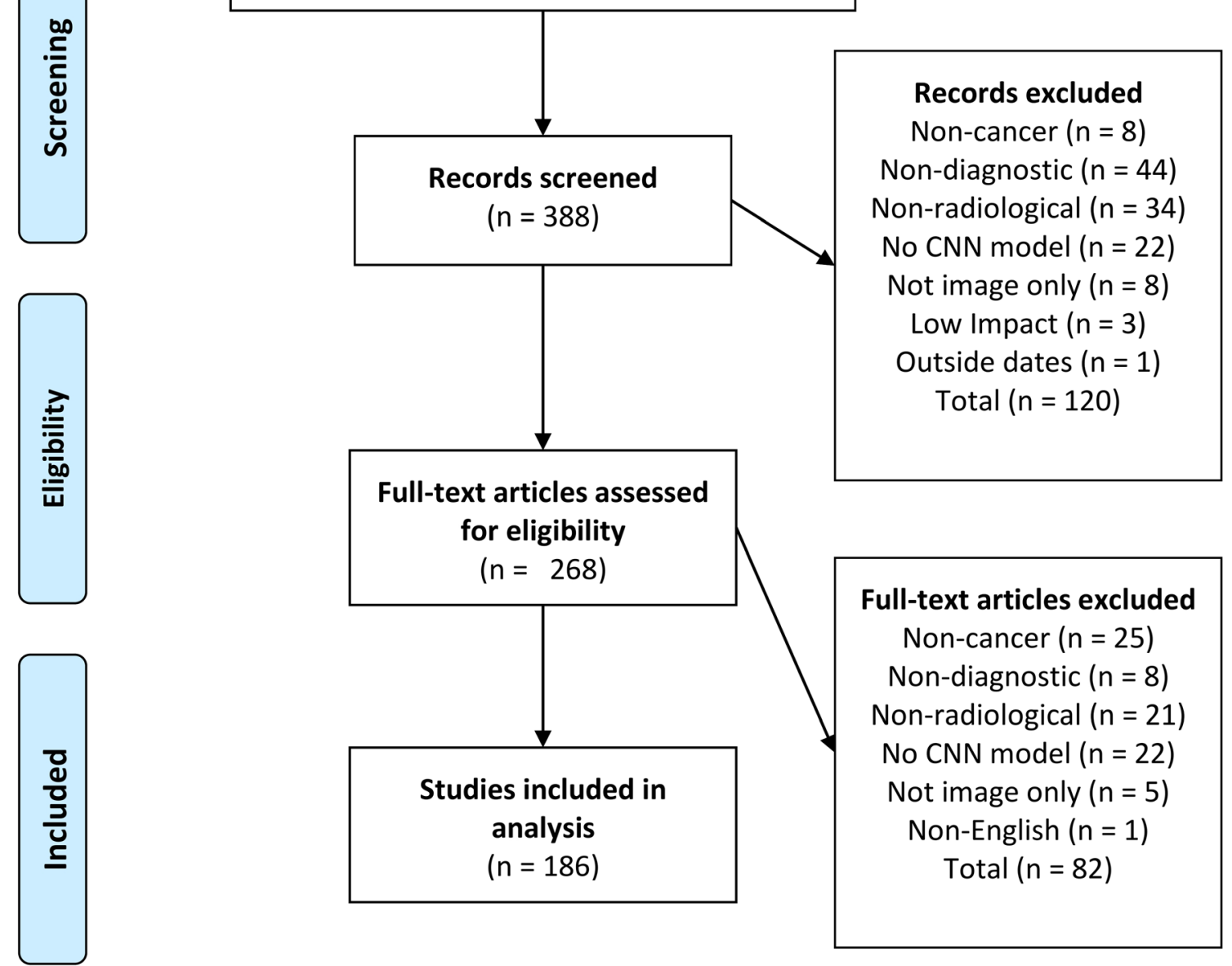

\section{Full-text articles excluded}

Non-cancer $(n=25)$

Non-diagnostic $(n=8)$

Non-radiological $(n=21)$

No CNN model $(n=22)$

Not image only $(n=5)$

Non-English $(n=1)$

Total $(n=82)$

Fig. 1 Flow diagram of literature search process

presented a structured abstract (131/186, 95\% CI 63-77\%). Ninety-eight percent included scientific and clinical background (183/186, 95\% CI 95-100\%). Although 92\% of studies stated objectives $(171 / 186,95 \%$ CI $87-95 \%)$, only $4 \%$ included explicit hypotheses (8/186, 95\% CI 2-8\%).

\section{Study design}

Thirty-three percent of studies documented a retrospective or prospective timeframe $(62 / 186,95 \%$ CI $27-41 \%)$. Of these, $87 \%$ were retrospective $(54 / 62$, 95\% CI $81-95 \%)$, 

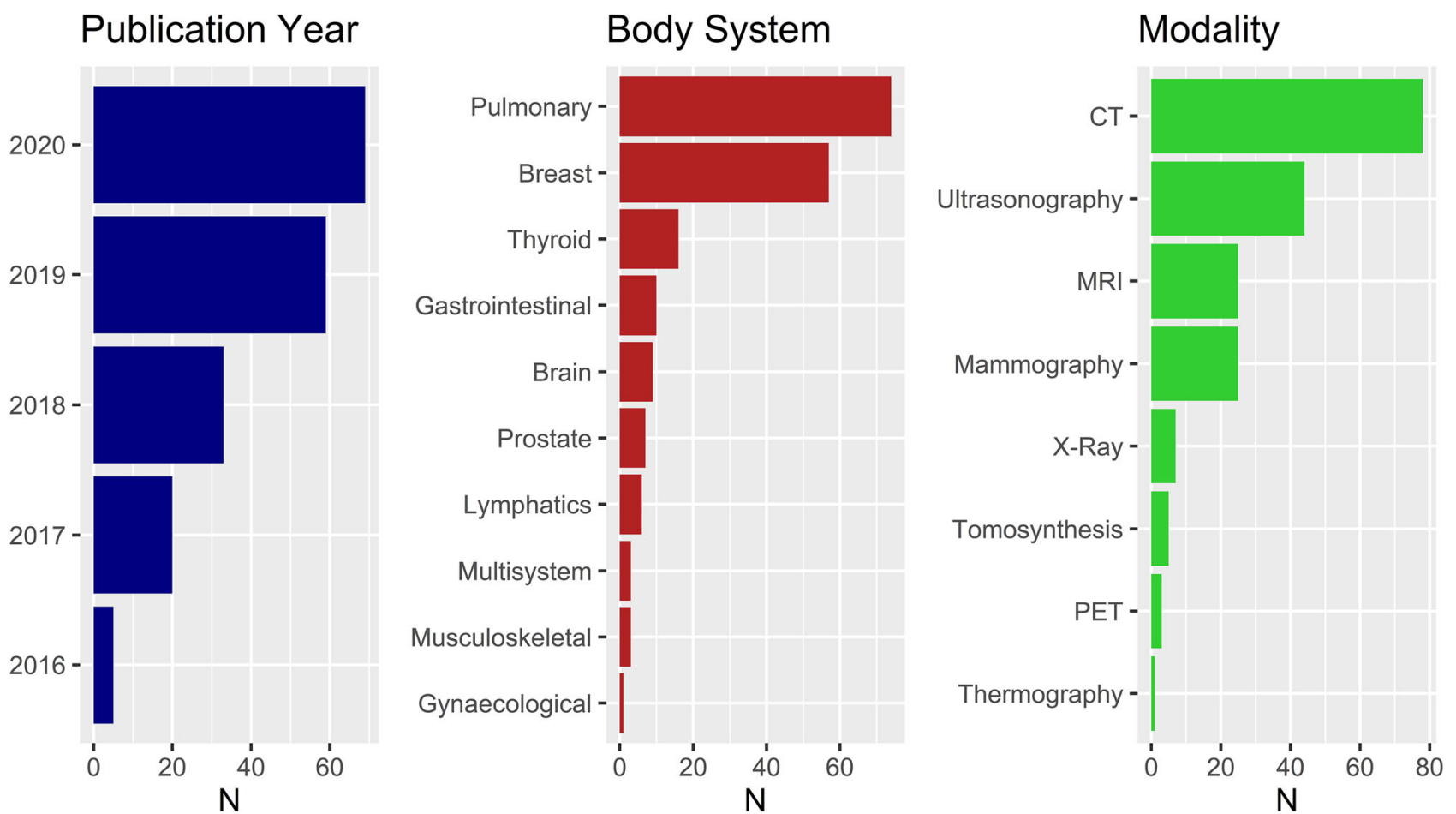

Fig. 2 Distribution of included articles. Left: study publication year. Middle: body system imaged. Right: imaging modality employed

$10 \%$ were prospective $(6 / 62,95 \%$ CI $3-18 \%)$ and $3 \%$ used both retrospective and prospective data $(2 / 62,95 \%$ CI $0-11 \%)$. Twenty-five percent of studies specified a goal $(47 / 186,95 \%$ CI 19-32\%).

\section{Data}

Ninety-four percent of studies documented their data sources (174/186, 95\% CI 89-97\%). Of these, 45\% used publicly available datasets only (79/174, 95\% CI 38-53\%), 49\% used local data only $(85 / 174,95 \%$ CI $41-57 \%)$ and $6 \%$ combined public datasets with locally collected data $(10 / 174,95 \%$ CI 0 $14 \%)$. Seventy-two percent of studies documented the centres from which the data was sourced $(134 / 185,95 \%$ CI $65-79 \%)$. Of these, $50 \%$ used data from multiple centres $(67 / 135,95 \%$ CI 41-58\%). Fifty-one percent of studies detailed the imaging equipment used $(94 / 186,95 \%$ CI $43-58 \%)$. Of these, $53 \%$ employed equipment from multiple vendors $(50 / 94,95 \%$ CI 43-64\%). Image acquisition parameters were documented in $37 \%$ of studies $(69 / 186,95 \%$ CI $30-44 \%)$. Amongst studies which collected local data, $83 \%$ documented institutional review board approval (79/95, 95\% CI 74-90\%) and $26 \%$ documented participant consent $(25 / 95,95 \%$ CI $18-36 \%)$. In studies of publicly available data, $9 \%$ documented institutional review board approval $(7 / 79,95 \%$ CI $4-17 \%)$ and $8 \%$ documented participant consent $(6 / 79,95 \%$ CI 3-16\%). Twentyseven percent of studies documented eligibility criteria for their data $(50 / 186,95 \%$ CI $21-34 \%)$.
Pre-processing was documented in $69 \%$ of studies (128/ $186,95 \%$ CI $62-75 \%$ ), though only $53 \%$ provided a reproducible methodology $(98 / 186,95 \%$ CI 46-60\%). Data subsetting was applied in $42 \%$ of studies $(78 / 186,95 \% \mathrm{CI}$ $35-49 \%$ ), of which $95 \%$ included methods $(74 / 78,95 \% \mathrm{CI}$ 87-99\%). As per our inclusion criteria, all studies employed convolutional neural network models, which define predictor features autonomously. We also required an outcome in the domain of radiological cancer diagnosis. Therefore, relevant data elements were defined in $100 \%$ of included studies (186/ 186 , 95\% CI 98-100\%). Nineteen percent of studies performing local data collection documented data anonymisation $(18 / 95,95 \%$ CI $12-28 \%)$, though only $3 \%$ detailed the methodology (3/95, 95\% CI 0-11\%). Eightyfour percent of studies performing local data collection documented data anonymisation, institutional review board approval or both $(80 / 95,95 \%$ CI $75-91 \%)$. Three percent of studies of publicly available data documented data anonymisation (3/91, 95\% CI 1-9\%), and none detailed the methodology. Missing data procedures were documented in $17 \%$ of studies $(31 / 186,95 \%$ CI $12-23 \%)$. Case exclusion was the only strategy employed to manage missing data in these studies $(31 / 31,95 \%$ CI 89-100\%).

\section{Ground truth}

Compliance for items 14-27 is shown in Fig. 4. Twenty-five percent of studies defined the reference standard or used a 


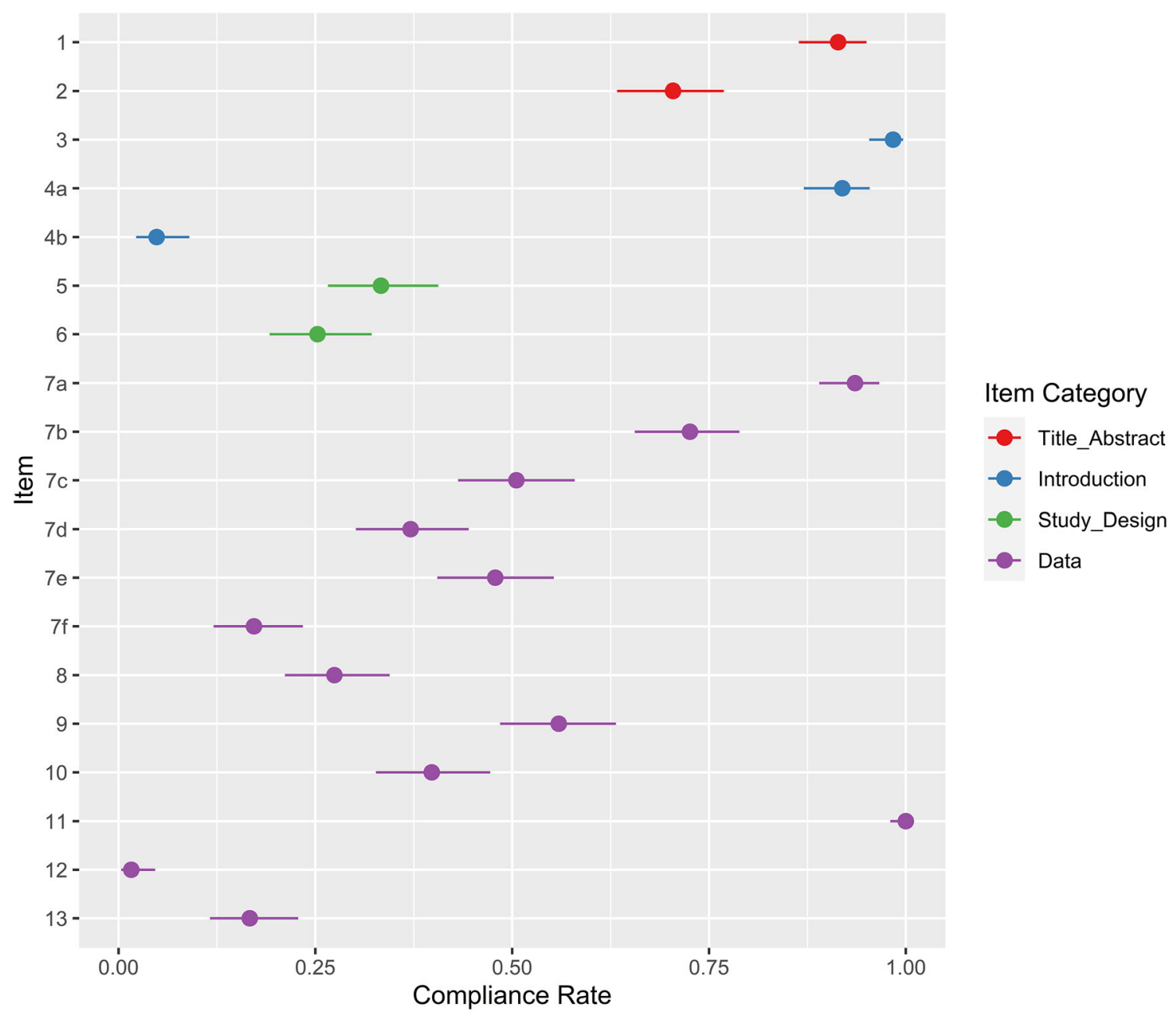

Fig. 3 Compliance with CLAIM items 1-13. Compliance rate is defined as the proportion of articles subject to that item which satisfy it. Exemptions are provided in Table 1. Point estimates and 95\% confidence intervals are reported

Common Data Element such as the American College of Radiology Reporting and Data Systems (46/186, 95\% CI $19-32 \%)$. Three percent of studies provided rationale for the reference standard $(5 / 186,95 \%$ CI $1-6 \%)$. However, $50 \%$ employed definitive clinical standards such as histopathology, case follow-up or definitive imaging modalities $(93 / 186,95 \%$ CI $43-57 \%)$. Of these, $77 \%$ used histopathology $(72 / 93,95 \%$ CI $70-86 \%), 15 \%$ used histopathology in combination with follow-up imaging (14/93, 95\% CI 8-23\%) and 2\% used histopathology in combination with definitive imaging (2/93, 95\% CI 0-10\%). Four percent used follow-up only (14/93, 95\% CI $8-23 \%$ ), and $1 \%$ used definitive imaging only $(1 / 93,95 \%$ CI $0-9 \%)$.

Forty percent of studies documented image annotation by a radiologist with relevant subspecialist expertise (75/186, 95\% CI 33-48\%). A further 32\% documented annotation by a radiologist with unspecified expertise $(60 / 186,95 \%$ CI $25-$ $40 \%)$ and $4 \%$ used other clinicians $(8 / 186,95 \%$ CI $0-12 \%)$.
Of the studies which utilised histopathological ground truth, $8 \%$ specified annotation by a pathologist with relevant subspecialist experience $(7 / 88,95 \%$ CI 3-16\%). Twelve percent of studies documented the software tools used for image annotation $(22 / 186,95 \%$ CI 8-17\%). Eighteen percent of studies provided inter-rater or intra-rater variability statistics $(34 / 186$, 95\% CI $13-25 \%$ ), and $27 \%$ provided their aggregation strategy $(50 / 186,95 \%$ CI $21-34 \%)$, though only $16 \%$ provided both $(30 / 186,95 \%$ CI $11-22 \%)$.

\section{Data partitions}

Eighty-seven percent of studies reported the number of images modelled (161/186, 95\% CI $81-91 \%)$, though only $1 \%$ provided a power calculation $(1 / 186,95 \%$ CI $0-3 \%)$. Seventytwo percent specified the number of study participants in their dataset (133/186, 95\% CI 64-78\%). Of these, a median of 367 participants were included (IQR 172-1000). Seven studies 


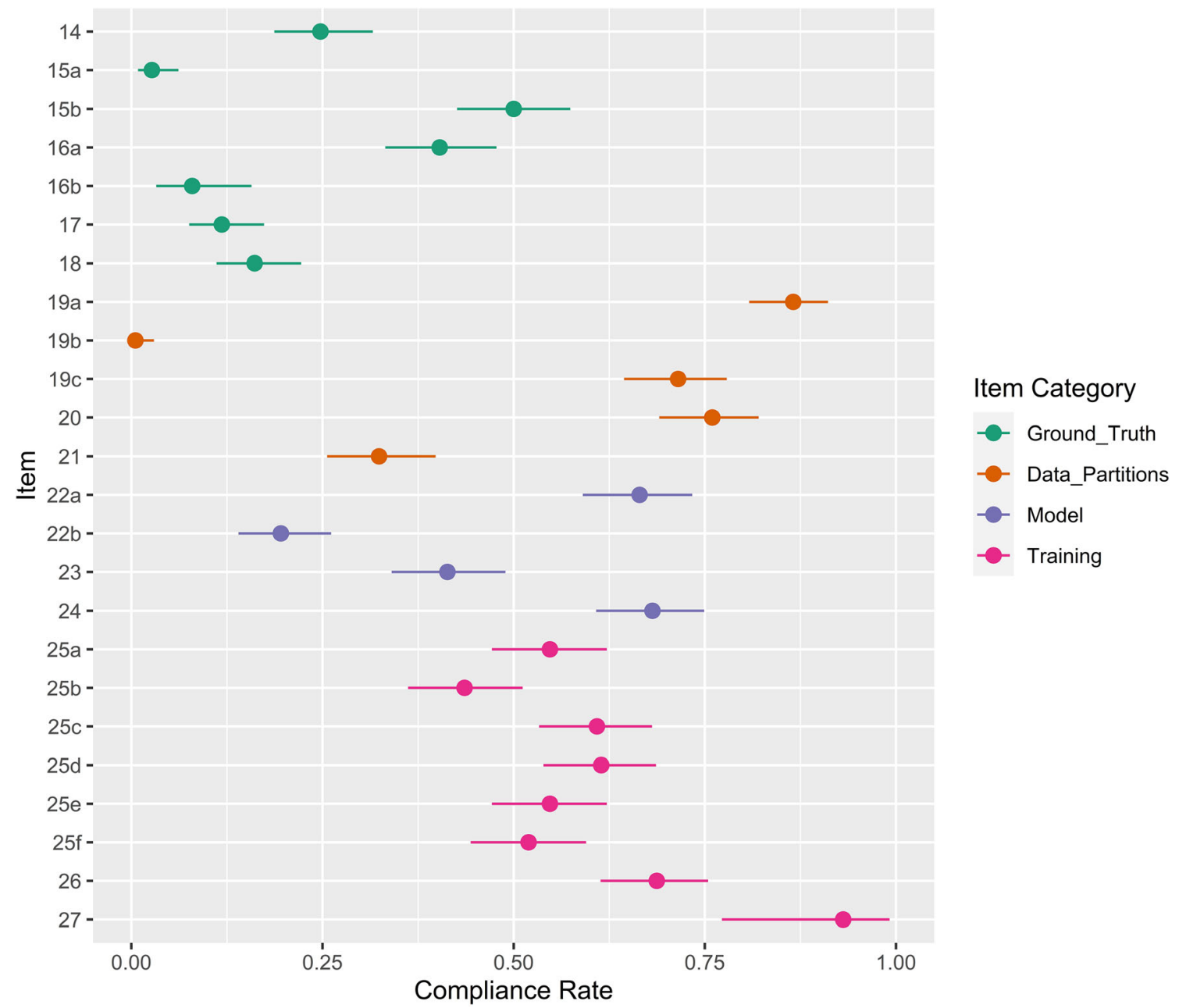

Fig. 4 Compliance with CLAIM items 14-27. Compliance rate is defined as the proportion of articles subject to that item which satisfy it. Exemptions are provided in Table 1. Point estimates and $95 \%$ confidence intervals are reported

served only to validate existing models and were exempted from criteria pertaining to model development and data partitioning. Seventy-six percent of modelling studies defined data partitions and their proportions $(136 / 179,95 \%$ CI 69 $82 \%$ ), though $32 \%$ specified the level of partition disjunction $(58 / 179,95 \%$ CI $26-40 \%)$.

\section{Model}

Sixty-six percent of modelling studies provided a detailed model description (119/179, 95\% CI 59-73\%) and $20 \%$ of modelling studies provided access to source code $(35 / 179$, 95\% CI 14-26\%). Sixty-eight percent documented the development software $(122 / 179,95 \%$ CI $61-75 \%)$, though only $41 \%$ included the software version $(74 / 179,95 \%$ CI $34-$ 49\%). Sixty-eight percent of modelling studies reported model initialisation parameters $(122 / 179,95 \%$ CI $61-75 \%)$. Of these, 52\% employed transfer learning $(93 / 179,95 \%$ CI 44 $59 \%$ ) and $3 \%$ compared transfer learning with random initialisation $(4 / 124,95 \%$ CI $1-8 \%)$.

\section{Training}

Sixty-five percent of modelling studies reported data augmentation (117/179, 95\% CI 58-72\%), though only 54\% documented reproducible methodology $(96 / 179,95 \%$ CI $46-$ $61 \%)$. Sixty-one percent of modelling studies documented the optimisation algorithm $(109 / 179,95 \%$ CI $53-68 \%), 61 \%$ documented learning rate $(110 / 179,95 \%$ CI $54-69 \%), 44 \%$ documented loss function $(78 / 179,95 \%$ CI $36-51 \%)$ and $52 \%$ documented batch size $(93 / 179,95 \%$ CI $44-59 \%)$. Model selection strategies were documented in $69 \%$ of modelling studies $(123 / 179,95 \%$ CI $61-75 \%)$. Of 30 studies which employed model ensembling, $93 \%$ reported their aggregation methodology (28/30, 95\% CI 78-99\%).

\section{Evaluation}

Compliance with CLAIM items $28-42$ is shown in Fig. 5. Fifty-five percent of studies defined performance metrics (103/186, 95\% CI 48-63\%) and 36\% provided some rationale 


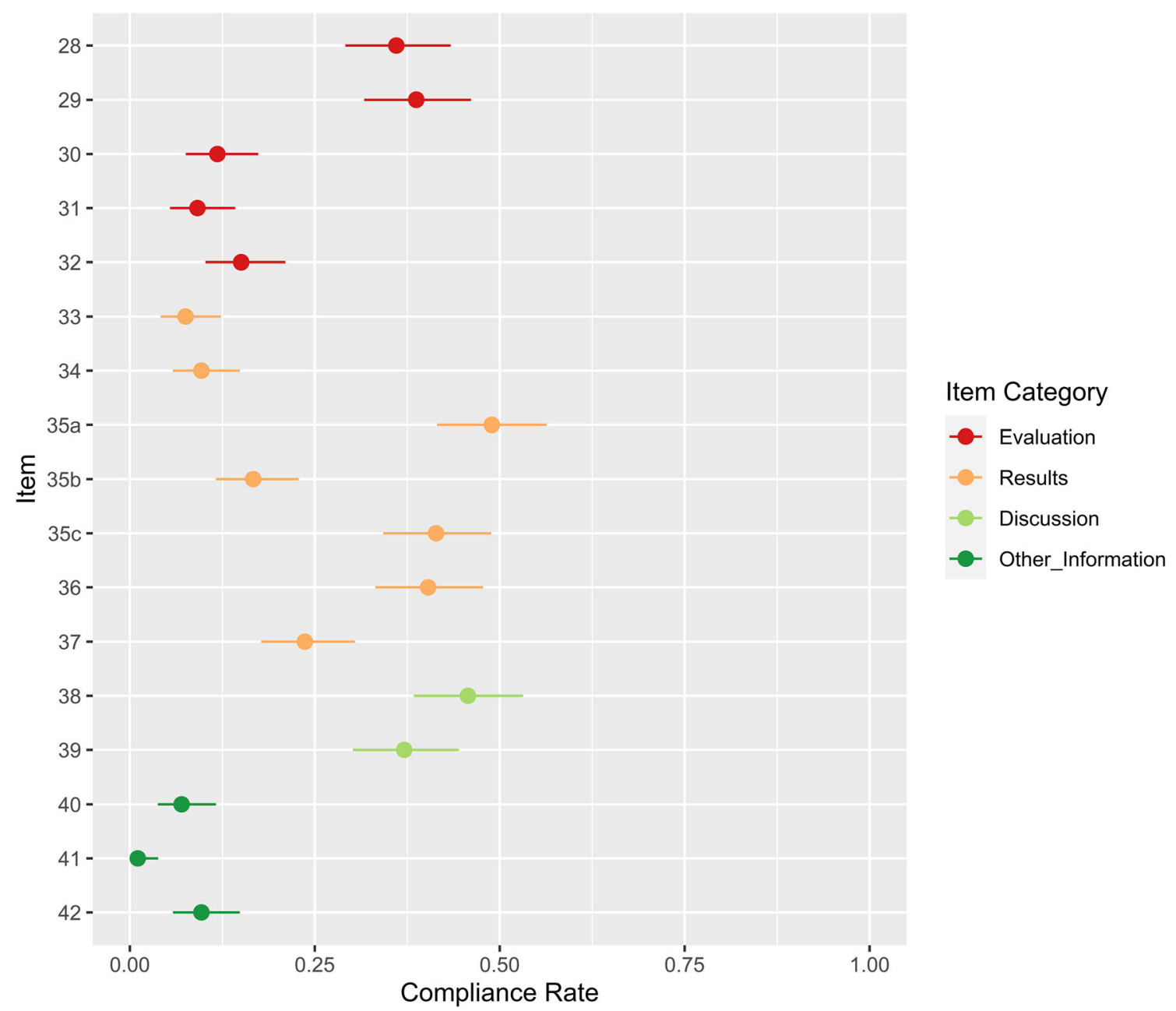

Fig. 5 Compliance with CLAIM items 28-42. Compliance rate is defined as the proportion of articles subject to that item which satisfy it. Exemptions are provided in Table 1. Point estimates and $95 \%$ confidence intervals are reported

for these (67/186, 95\% CI 29-43\%). Statistical significance of results was reported with methodology in $61 \%$ of studies (114/186, 95\% CI 54-68\%) and without methodology in 97\% (181/186, 95\% CI 94-99\%). Twelve percent of studies evaluated model robustness (22/186, 95\% CI 8-17\%). Although $25 \%$ of studies attempted some interpretation of the model (47/186, 95\% CI 19-32\%), only $9 \%$ provided validated methodology for their procedure $(17 / 186,95 \%$ CI 5 $14 \%)$.

Forty-nine percent of studies assessed model performance on test data which was explicitly disjunct from training and validation data $(91 / 186,95 \%$ CI $42-57 \%)$. Forty-five percent of studies mentioned only two data partitions $(83 / 186,95 \%$ CI $38-53 \%$ ); their reported results may have represented validation rather than test performance. A further $6 \%$ failed to document any data partitions (12/186, 95\% CI 0-14\%); their reported results may have represented training performance. Forty-one percent of studies benchmarked models against other computational methods $(77 / 186,95 \%$ CI $34-49 \%)$. Seventeen percent of studies benchmarked their model against radiologists with relevant subspecialist expertise (31/186, 95\% CI 11-23\%). Five percent of studies benchmarked their model against radiologists without specifying expertise (9/186, 95\% CI $0-11 \%)$ and $1 \%$ employed other clinicians $(1 / 186,95 \%$ CI $0-7 \%)$.

\section{Results}

Case flow diagrams were provided in $8 \%$ of studies $(14 / 186$, 95\% CI 4-12\%). Thirty-one percent of studies reported demographic and clinical characteristics of their population (58/ $186,95 \%$ CI $25-38 \%$ ). However, only $10 \%$ described separate distributions for each data partition (18/186, 95\% CI 6$15 \%)$. Fifteen percent of studies reported performance metrics on test data from another institution $(28 / 186,95 \%$ CI $8-23 \%)$. Thirty-four percent used test data from the same institution (63/186, 95\% CI 27-41\%). Diagnostic accuracy was reported with confidence intervals in $40 \%$ of studies $(75 / 186,95 \% \mathrm{CI}$ $33-48 \%)$. Twenty-four percent of studies discussed misclassified examples (44/186, 95\% CI 18-30\%). 


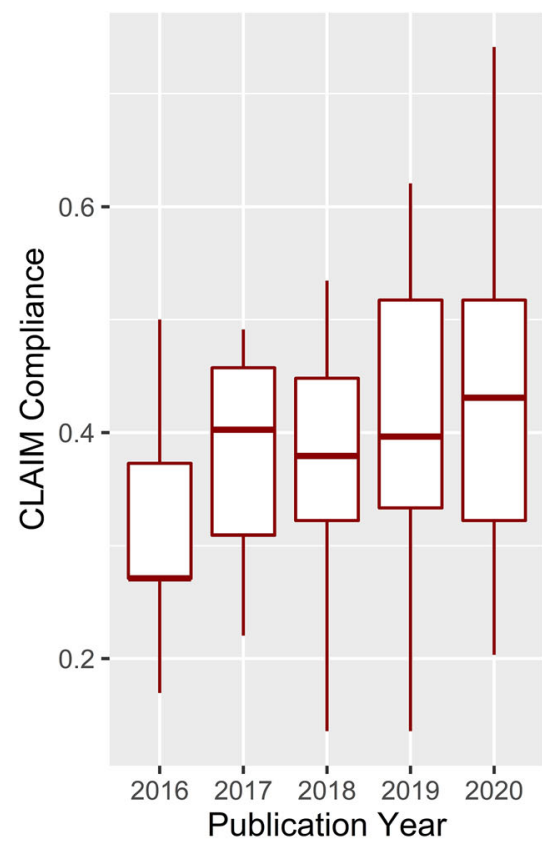

Fig. 6 Left: CLAIM compliance over time. Compliance was defined per article by the proportion of applicable items satisfied. Boxplot centrelines indicate median annual compliance. Hinges indicate first and third quartiles. Whiskers indicate maxima and minima. Middle: CLAIM compliance and journal $\mathrm{H}$-index for each article. Right: CLAIM
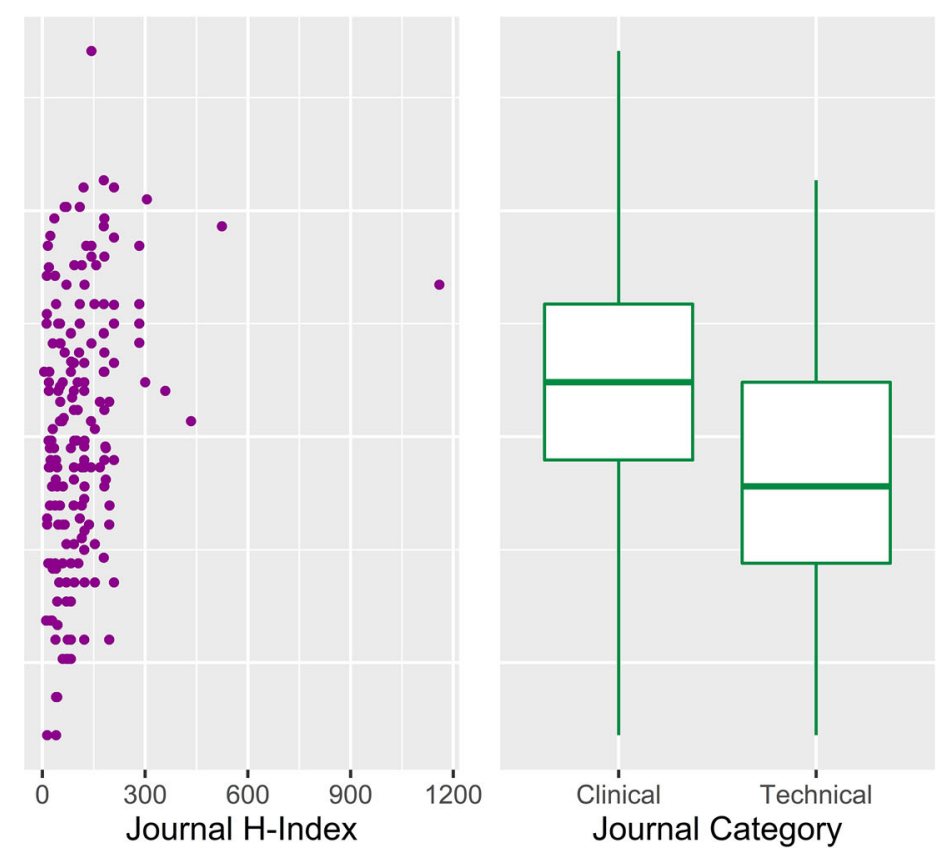

compliance in clinical journals and technical journals. Journals were categorised as either "clinical" or "technical" according to the journal name - names containing any term related to computer science, artificial intelligence or machine learning were assigned the "technical" category. The remaining journals were assigned the "clinical" category

\section{Discussion}

Forty-six percent of studies discussed limitations $(85 / 186$, 95\% CI 38-53\%) and 37\% clinical implications of their findings $(69 / 186,95 \%$ CI $30-44 \%)$.

\section{Other information}

Study registration numbers were provided in $7 \%$ of studies $(13 / 186,95 \%$ CI $3.8-11.7 \%)$, and study protocols in $1 \%$ $(2 / 186,95 \%$ CI $0.1-3.8 \%)$. Funding was documented in $65 \%$ of studies (121/186, 95\% CI $57.7-71.9 \%)$ though only $3 \%$ included the role of the funding institution $(6 / 186,95 \%$ CI $0.0-10.9 \%$ ). A further $6 \%$ of studies stated that they did not receive funding (12/186, 95\% CI 0.0-14.1\%). Compliance for items $1-42$ is provided in Supplementary Table 3.

\section{Overall CLAIM compliance}

Median CLAIM compliance was 0.40 (IQR 0.33-0.49). Compliance correlated positively with publication year $(\rho=$ $0.15, p=.04)$ and journal H-index $(\rho=0.27, p<.001)$. Clinical journals demonstrated higher mean compliance than technical journals $(0.44$ vs. $0.37, p<.001)$. Compliance distribution is visualised with respect to publication year, journal H-index and journal category in Fig. 6 .

\section{Discussion}

Radiological AI is undergoing a development phase, reflected in growing annual publication volume and recognition by clinical researchers [33-37]. To safely harness the potential of new methodologies, clinicians have called for realistic, reproducible and ethical research practices [1,38-44]. The CLAIM guidance sets stringent standards for research in this domain, amalgamating the technical requirements of the statistical learning field $[9,45]$ with the practicalities of clinical research $[1,2,15,46]$. We observed improvements in documentation standards improved over time, a finding concurrent with previous reviews of AI research [43, 45]. Compliance was highest in impactful clinical journals, demonstrating the value of design and reporting practices at peer review.

A key opportunity for improvement is model testing, addressed by items 20,21, 32 and 35. Documentation should specify three disjoint data partitions for CNN modelling (which may be resampled with cross-validation or bootstrapping). Training data is used for model learning, validation data for model selection and test data to assess performance of a finalised model [47, 48]. Half of studies documented two or less partitions - in these cases, results may have represented validation or even training performance. Where data partitions were not disjoint on perpatient basis, data leakage may have occurred despite partitioned model testing. These scenarios bias generalisability metrics 
optimistically. Some multi-centre studies partitioned data at the patient level rather than the institutional level, missing an opportunity to evaluate inter-institution generalisability.

Evidently, CLAIM has also introduced requirements which depart from current norms. Few studies satisfied item 12, which requires the documentation of data anonymisation methods, an issue which has developed with image recognition capabilities $[41,49,50]$. This requirement may have previously been relaxed for studies of publicly available data or those which documented institutional review board approval, as either case suggests previous certification of data governance procedures. The spirit of the CLAIM guidance is obviation of such assumptions with clear documentation, promoting a culture of research transparency. In many such cases, the burden of improved compliance is minimal, mandating only the documentation of additional information.

Our findings concur with previous reviews of design and reporting standards in both clinical and general-purpose AI research. A review of studies benchmarking AI against radiologists identified deficient documentation of data availability, source code, eligibility and study setting [38]. Reviews of TRIPOD adherence in multivariate diagnostic modelling found deficient model assessment and data description [12, $51,52]$. Reviews of reproducibility in AI research have reported insufficient documentation of data availability, source code, protocols and study registration [43, 45, 53]. Many commentators have advocated for transparency in clinical AI research [19, 38, 40, 42, 43, 53, 54].

We note several limitations to this systematic review. First, as scope was limited to studies published in English, findings were susceptible to language bias. Second, although reporting standards were directly measurable, items relating to study design were only measurable if reported. Consequently, design compliance may have been underestimated in poorly reported studies. This is a general limitation of reviews in this field. Third, articles were read sequentially and therefore readers were potentially susceptible to anchoring bias. The effect of anchoring on the trend and subgroup analyses was minimised by randomisation of the reading order.

\section{Conclusions}

Design and reporting standards have improved in CNN research for radiological cancer diagnosis, though many opportunities remain for further progress. The CLAIM guidance sets a high standard for this developing field, consolidating clinical and technical research requirements to enhance the quality of evidence. Our data supports the need for integration of CLAIM guidance into the design and reporting of CNN studies for radiological cancer diagnosis.
Supplementary Information The online version contains supplementary material available at https://doi.org/10.1007/s00330-021-07881-2.

Funding The authors acknowledge financial support from the UK Research \& Innovation London Medical Imaging and Artificial Intelligence Centre and Wellcome Trust EPSRC Centre for Medical Engineering at King's College London (WT203148/Z/16/Z).

\section{Declarations}

Guarantor The scientific guarantor of this publication is Vicky Goh (Vicky.goh@kcl.ac.uk).

Conflict of interest The authors of this manuscript declare no relationships with any companies whose products or services may be related to the subject matter of the article.

Statistics and biometry No complex statistical methods were necessary for this paper.

Informed consent Written informed consent was not required for this study because this was a systematic review using published studies in the literature but not analysing specific human subjects.

Ethical approval Institutional review board approval was not required because this was a systematic review using published studies in the literature but not analysing specific human subjects.

\section{Methodology}

- Retrospective

- Multicentre study

Open Access This article is licensed under a Creative Commons Attribution 4.0 International License, which permits use, sharing, adaptation, distribution and reproduction in any medium or format, as long as you give appropriate credit to the original author(s) and the source, provide a link to the Creative Commons licence, and indicate if changes were made. The images or other third party material in this article are included in the article's Creative Commons licence, unless indicated otherwise in a credit line to the material. If material is not included in the article's Creative Commons licence and your intended use is not permitted by statutory regulation or exceeds the permitted use, you will need to obtain permission directly from the copyright holder. To view a copy of this licence, visit http://creativecommons.org/licenses/by/4.0/.

\section{References}

1. Bluemke DA, Moy L, Bredella MA et al (2019) Assessing radiology research on artificial intelligence: a brief guide for authors, reviewers, and readers-from the Radiology Editorial Board. Radiology:192515. https://doi.org/10.1148/radiol.2019192515

2. Bi WL, Hosny A, Schabath MB et al (2019) Artificial intelligence in cancer imaging: clinical challenges and applications. CA Cancer J Clin 69:127-157. https://doi.org/10.3322/caac.21552

3. Mendelson EB (2019) Artificial intelligence in breast imaging: potentials and limitations. AJR Am J Roentgenol 212:293-299

4. Gilbert FJ, Smye SW, Schönlieb CB (2020) Artificial intelligence in clinical imaging: a health system approach. Clin Radiol 75:3-6 
5. O’Regan DP (2020) Putting machine learning into motion: applications in cardiovascular imaging. Clin Radiol 75:33-37

6. Liu X, Faes L, Kale AU et al (2019) A comparison of deep learning performance against health-care professionals in detecting diseases from medical imaging: a systematic review and meta-analysis. Lancet Digit Health 1:e271-e297. https://doi.org/10.1016/S25897500(19)30123-2

7. Lecun Y, Bengio Y, Hinton G (2015) Deep learning. Nature 521: 436-444

8. Erickson BJ, Korfiatis P, Kline TL et al (2018) Deep learning in radiology: does one size fit all? J Am Coll Radiol 15:521-526. https://doi.org/10.1016/j.jacr.2017.12.027

9. Cawley GC, Talbot NLC (2010) On over-fitting in model selection and subsequent selection bias in performance evaluation. J Mach Learn Res 11:2079-2107

10. Yasaka K, Akai H, Kunimatsu A et al (2018) Deep learning with convolutional neural network in radiology. Jpn J Radiol 36:257-272

11. Kelly CJ, Karthikesalingam A, Suleyman M et al (2019) Key challenges for delivering clinical impact with artificial intelligence. BMC Med 17:195. https://doi.org/10.1186/s12916-019-1426-2

12. Yusuf M, Atal I, Li J et al (2020) Reporting quality of studies using machine learning models for medical diagnosis: a systematic review. BMJ Open 10:e034568. https://doi.org/10.1136/bmjopen-2019034568

13. Simera I, Altman DG, Moher D et al (2008) Guidelines for reporting health research: the EQUATOR network's survey of guideline authors. PLoS Med 5:0869-0874. https://doi.org/10. 1371/journal.pmed.0050139

14. Pandis N, Fedorowicz Z (2011) The international EQUATOR network: enhancing the quality and transparency of health care research. J Appl Oral Sci:19. https://doi.org/10.1590/S167877572011000500001

15. Bossuyt PM, Reitsma JB, Bruns DE et al (2015) STARD 2015: an updated list of essential items for reporting diagnostic accuracy studies1. Radiology 277:826-832. https://doi.org/10.1148/radiol. 2015151516

16. Vandenbroucke JP, Von Elm E, Altman DG et al (2007) Strengthening the Reporting of Observational Studies in Epidemiology (STROBE): explanation and elaboration. PLoS Med 4:1628-1654. https://doi.org/10.1371/journal.pmed.0040297

17. Schulz KF, Altman DG, Moher D (2010) CONSORT 2010 statement: updated guidelines for reporting parallel group randomised trials. BMJ 340:698-702. https://doi.org/10.1136/bmj.c332

18. Mongan J, Moy L, Kahn CE (2020) Checklist for Artificial Intelligence in Medical Imaging (CLAIM): a guide for authors and reviewers. Radiol Artif Intell 2:e200029. https://oi.org/10.1148/ ryai.2020200029

19. Rivera SC, Liu X, Chan A-W et al (2020) Consensus statement Guidelines for clinical trial protocols for interventions involving artificial intelligence: the SPIRIT-AI extension The SPIRIT-AI and CONSORT-AI Working Group*, SPIRIT-AI and CONSORT-AI Steering Group and SPIRIT-AI and CONSORT-AI Cons. Nat Med 26:1351-1363. https://doi.org/10.1038/s41591-020-1037-7

20. Norgeot B, Quer G, Beaulieu-jones BK et al (2020) Minimum information about clinical artificial intelligence modeling: the MI-CLAIM checklist. Nat Med 26. https://doi.org/10.1038/s41591-020-1042-x

21. Sounderajah V, Ashrafian H, Aggarwal R et al (2020) Developing specific reporting guidelines for diagnostic accuracy studies assessing AI interventions: The STARD-AI Steering Group. Nat Med 26:807-808. https://doi.org/10.1038/s41591-020-0941-1

22. Hinton GE, Srivastava N, Krizhevsky A, Sutskever I, Salakhutdinov RR (2012) Improving neural networks by preventing co-adaptation of feature detectors. arXiv:12070580v1

23. Soffer S, Ben-Cohen A, Shimon O et al (2019) Convolutional neural networks for radiologic images: a radiologist's guide. Radiology 290: 590-606
24. Litjens G, Kooi T, Bejnordi BE et al (2017) A survey on deep learning in medical image analysis. Med Image Anal 42:60-88

25. Whiting P, Rutjes AWS, Reitsma JB et al (2003) The development of QUADAS: a tool for the quality assessment of studies of diagnostic accuracy included in systematic reviews. BMC Med Res Methodol 3:1-13

26. Team R Development Core (2019) A language and environment for statistical computing. R Found Stat Comput 2. https://www.R-project. org

27. SCImago (n.d.). SJR - SCImago Journal \& Country Rank [Portal]. Retrieved from http://www.scimagojr.com/. Accessed 14 Dec 2020

28. RStudio Team (2020) RStudio: integrated development for R. Version 1.1.463. http://www.rstudio.com/

29. Clopper CJ, Pearson ES (1934) The use of confidence or fiducial limits illustrated in the case of the binomial. Biometrika 26:404. https://doi.org/10.2307/2331986

30. Sison CP, Glaz J (1995) Simultaneous confidence intervals and sample size determination for multinomial proportions. J Am Stat Assoc 90:366-369. https://doi.org/10.1080/01621459.1995.10476521

31. Signorell A et al. (2020) DescTools: Tools for Descriptive Statistics. R package version 0.99.37. https://cloud.rproject.org/ web/packages/DescTools/citation.html

32. Heus P, Damen JAAG, Pajouheshnia R et al (2019) Uniformity in measuring adherence to reporting guidelines: the example of TRIPOD for assessing completeness of reporting of prediction model studies. BMJ Open 9. https://doi.org/10.1136/bmjopen-2018-025611

33. Recht MP, Dewey M, Dreyer K et al (2020) Integrating artificial intelligence into the clinical practice of radiology: challenges and recommendations. Eur Radiol:1-9. https://doi.org/10.1007/s00330020-06672-5

34. Hosny A, Parmar C, Quackenbush J et al (2018) Artificial intelligence in radiology. Nat Rev Cancer 18:500-510. https://doi.org/10. 1038/s41568-018-0016-5

35. Killock D (2020) AI outperforms radiologists in mammographic screening. Nat Rev Clin Oncol 17:134. https://doi.org/10.1038/ s41571-020-0329-7

36. Bluemke DA (2018) Radiology in 2018: are you working with AI or being replaced by AI? Radiology 287:365-366

37. Davenport T, Kalakota R (2019) The potential for artificial intelligence in healthcare. Future Healthc J 6:94-98. https://doi.org/10. 7861/futurehosp.6-2-94

38. Nagendran M, Chen Y, Lovejoy CA et al (2020) Artificial intelligence versus clinicians: systematic review of design, reporting standards, and claims of deep learning studies in medical imaging. BMJ 368:m689. https://doi.org/10.1136/bmj.m689

39. Kitamura FC, Pan I, Kline TL (2020) Reproducible artificial intelligence research requires open communication of complete source code. Radiol Artif Intell 2:e200060. https://doi.org/10.1148/ryai. 2020200060

40. Geis JR, Brady AP, Wu CC et al (2019) Ethics of artificial intelligence in radiology: summary of the joint European and North American multisociety statement. J Am Coll Radiol 16:15161521. https://doi.org/10.1016/j.jacr.2019.07.028

41. Jaremko JL, Azar M, Bromwich R et al (2019) Canadian Association of Radiologists white paper on ethical and legal issues related to artificial intelligence in radiology. Can Assoc Radiol J 70:107-118. https://doi.org/10.1016/j.carj.2019.03.001

42. Haibe-Kains B, Adam GA, Hosny A et al (2020) Transparency and reproducibility in artificial intelligence. Nature 586:E14-E16. https://doi.org/10.1038/s41586-020-2766-y

43. Wallach JD, Boyack KW, Ioannidis JPA (2018) Reproducible research practices, transparency, and open access data in the biomedical literature, 2015-2017. PLoS Biol 16. https://doi.org/10.1371/ journal.pbio. 2006930 
44. Vollmer S, Mateen BA, Bohner G et al (2020) Machine learning and artificial intelligence research for patient benefit: 20 critical questions on transparency, replicability, ethics, and effectiveness. BMJ 368. https://doi.org/10.1136/bmj.16927

45. Gundersen OE, Kjensmo S (2018) State of the art: reproducibility in artificial intelligence. 32nd AAAI Conf Artif Intell AAAI 20181644 1651

46. Lambin P, Leijenaar RTH, Deist TM et al (2017) Radiomics: the bridge between medical imaging and personalized medicine. Nat Rev Clin Oncol 14:749-762

47. Ripley BD (2014) Pattern recognition and neural networks. Pattern Recognit Neural Netw:1-403. https://doi.org/10.1017/ CBO9780511812651

48. Russell S, Norvig P (2010) Artificial intelligence: a modern approach, 3rd edn. Prentice Hall

49. Moore SM, Maffitt DR, Smith KE et al (2015) De-identification of medical images with retention of scientific research value. Radiographics 35:727-735. https://doi.org/10.1148/rg.2015140244

50. Schwarz CG, Kremers WK, Therneau TM et al (2019) Identification of anonymous MRI research participants with face- recognition software. N Engl J Med 381:1684-1686. https://doi. org $/ 10.1056 /$ nejmc 1908881

51. Heus P, Damen JAAG, Pajouheshnia R et al (2018) Poor reporting of multivariable prediction model studies: towards a targeted implementation strategy of the TRIPOD statement. BMC Med:16. https://doi.org/10.1186/s12916-018-1099-2

52. Wynants L, Van Calster B, Collins GS et al (2020) Prediction models for diagnosis and prognosis of covid-19: systematic review and critical appraisal. BMJ 369. https://doi.org/10.1136/bmj.m1328

53. Wright BD, Vo N, Nolan J et al (2020) An analysis of key indicators of reproducibility in radiology. Insights Imaging 11 . https://doi. org/10.1186/s13244-020-00870-x

54. Luo W, Phung D, Tran T et al (2016) Guidelines for developing and reporting machine learning predictive models in biomedical research: a multidisciplinary view. J Med Internet Res 18:e323. https://doi.org/10.2196/jmir.5870

Publisher's note Springer Nature remains neutral with regard to jurisdictional claims in published maps and institutional affiliations. 